\title{
Analytical Comparison of Antibody-drug Conjugates Based on Good Manufacturing Practice Strategies
}

\author{
Zhala TawfiQ,* Yutaka Matsuda,** Melody Jane Alfonso,* Colin Clancy,* Veronica Robles,* \\ Monica Leung, * and Brian A. Mendelsohn*ं
}

*Ajinomoto Bio-Pharma Services, 11040 Roselle Street, San Diego, CA 92121, United States

**Ajinomoto Co., Inc., 1-1 Suzuki-cho, Kawasaki, Kawasaki, Kanagawa 210-8681, Japan

\begin{abstract}
The production of antibody-drug conjugates (ADCs) has been in great demand in the field of cancer therapeutics. Although cysteine-based conjugation is the most common and well known process for producing ADCs, multiple analytical methods are required for accurate drug-antibody ratio (DAR) determination due to the heterogeneity of the ADCs. Here we report various analytical methods for DAR analysis of traditional cysteine-based ADCs; additionally, apply a good manufacturing practice (GMP) strategy to produce a four hundred milligram ADC batch for use in good laboratory practice (GLP) studies. The work described herein not only evaluates several analytical performances but also provides guidance for future phase appropriate ADC production while establishing a unique analytical strategy.
\end{abstract}

Keywords Antibody-drug conjugate, cysteine conjugation, good manufacturing practice, drug antibody ratio

(Received December 14, 2019; Accepted February 14, 2020; Advance Publication Released Online by J-STAGE April 24, 2020)

\section{Introduction}

Clinical trials for antibody drug conjugates (ADCs) have rapidly increased in recent years. To date, there are more than eighty ADCs in clinical development with five ADCs on the market. ${ }^{1-3}$

In accordance with the growth of the ADC field, analytical characterization continues to play a crucial role in the discovery, development, and manufacturing of ADCs. ${ }^{4,5}$ The FDA in particular, requires the pharmaceutical industry to carefully analyze antibody-based biopharmaceuticals, ${ }^{6,7}$ which remains a challenge due to the heterogeneity and structural complexity of the compounds. Several analytical methods have been established in an effort to address this issue. ${ }^{4,5}$ High performance liquid chromatography (HPLC) analysis is the most popular analytical approach for drug antibody ratio (DAR) characterization, but it is important to develop multiple methods for application at different project stages to ensure accuracy of DAR determination. Previously, we reported analytical comparison for site-specific ADCs using six analytical techniques to establish the appropriate analytical method for each phase in the project cycle. ${ }^{8}$ The results indicate the simple composition of a site-specific ADC is the preferred model for demonstrating new analytical techniques. However, comparative studies regarding multiple types of analysis for DAR determination using a single ADC batch are extremely limited in a peer-reviewed setting. ${ }^{8-12}$ Moreover, no examination to compare HPLC results among different ADCs prepared by various TCEP equivalents was reported to best of our knowledge. Investigation of TCEP titration is crucial to determine the

Z. T. and Y. M. contributed equally to this work.

† To whom correspondence should be addressed.

E-mail: Brian.Mendelsohn@US.AjiBio-Pharma.com appropriate equivalents to obtain cysteine-based ADCs with target DAR. Furthermore, different DAR results obtained from different analytical methods for single batch ADCs were observed in our previous investigation using site-specific ADCs. Cysteine-based ADCs, which are most commonly used for clinical trials, have more complicated structures than sitespecific ADCs, making it more difficult to get the actual DAR value by a single analysis. For this reason, analytical guidance to explain the TCEP dependency comparison of different analyses could be required.

In addition to analytical comparison, a good manufacturing practice (GMP) strategy is also required for robust ADC manufacturing to support use in clinical trials. Despite this, no reports in literature provided "GMP guidance" prior to our previous report. ${ }^{13}$ In 2019, our group reported a successful GMP strategy implementation for site-specific ADC preparation, ${ }^{13}$ however, this strategy still requires application to stochastic type ADCs, which use the same conjugation methodology as the ADCs currently on the market. Two ADCs (Adceteris, ${ }^{14}$ Polivy ${ }^{15}$ ) on the market are synthesized via the cysteine-based conjugation methodology, therefore cysteinebased conjugation is considered the gold standard technique for synthesizing a wide variety of ADCs. Taking that into consideration, the GMP strategy we previously established should be applied to cysteine type conjugation in an effort to provide guidance for the production of traditional ADCs.

In this report, we described analytical comparison using three different major techniques for DAR characterization and demonstrated the application of our GMP strategy to prepare a multi-hundred milligram cysteine-based ADC batch for use in good laboratory practice (GLP) studies. For this GLP study, we selected trastuzumab-MMAE with an approximate DAR of 4.0 as the target ADC material. Trastuzumab is a commonly used antibody for conjugation studies and relatively easy to obtain 

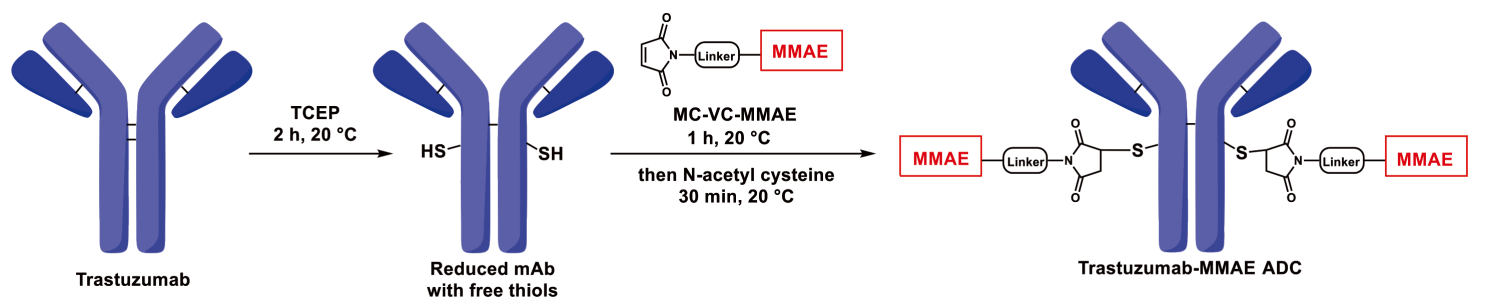

Fig. 1 Overview of the conjugation reaction.

from commercial resources, while MMAE was the most popular payload in the ADC field. Hence, trastuzumab-MMAE conjugates were considered to be ideal model ADCs for GLP studies.

These studies not only present an analytical strategy applicable to a wide variety of ADCs, but also serve as unique guidance for the analytical community aiming at clinical regulatory filings for biopharmaceutical compound manufacturing.

\section{Materials and Methods}

\section{Materials}

Human IgG1 trastuzumab (Herceptin) was purchased from Roche Pharmaceutical Company (Switzerland). Maleimide-C6valine-citrulline-monomethyl auristatin E (CAS\#: 646502-53-6; MC-VC-MMAE) was purchased from NJ Biopharmaceuticals LLC (USA). All other chemical reagents were acquired from Sigma-Aldrich (USA).

Synthetic procedure for cysteine-based ADC based on GMP strategy $y^{16}$

The commercially available trastuzumab (441 mg) was dissolved in water then buffer exchanged into conjugation buffer (55 mL, pH 7.5, $50 \mathrm{mM}$ PBS, $10 \mathrm{mM}$ EDTA) to prepare for the conjugation process. The reduction reaction began with the addition of 2.35 eq. Tris-(2-carboxyethyl)-phosphine hydrochloride (TCEP-HCl) to antibody and stirred mildly for $2 \mathrm{~h}$ at $20^{\circ} \mathrm{C}$. The resulting reaction mixture, dimethylacetamide (DMA) $(6 \% \mathrm{v} / \mathrm{v})$ and 6 eq. of MC-VC-MMAE were sequentially added and stirred mildly for $1 \mathrm{~h}$ at $20^{\circ} \mathrm{C}$. Unreacted drug linker was quenched with the addition of 25 eq. of $N$-acetyl-L-cysteine (NAC) and mixed for $35 \mathrm{~min}$ at $20^{\circ} \mathrm{C}$. The final mixture was purified using Centripure P100 desalting columns (purchased from emp Biotech LLC, USA) and eluted with $\mathrm{pH}$ 5.2, $20 \mathrm{mM}$ histidine, $5 \%$ trehalose.

Experimental procedure for thiol intermediate analysis by Ellman's assay

After completion of the reduction step, gel filtration of the reaction mixture was conducted to remove impurities and obtain a purified reduced antibody. The purified and reduced antibody was analyzed by Ellman's assay, as previously reported. ${ }^{17}$

\section{Instruments/analytical method}

Protein concentration was determined by the Slope Spectroscopy ${ }^{\circledR}$ method with a Solo-VPE system. ${ }^{17}$ Hydrophobic interaction chromatography (HIC) and reversed phase (RP)HPLC were performed as previously reported. ${ }^{13}$ Size exclusion chromatography (SEC) analysis was performed as previously reported. ${ }^{18}$

Endotoxin assay was performed by Endosafe-PTS system (Charles River, USA).
Table 1 Summary of three different analyses

\begin{tabular}{ccccc}
\hline Entry & $\begin{array}{c}\text { TCEP } \\
\text { equivalent }\end{array}$ & $\begin{array}{c}\text { DAR by } \\
\text { RP-HPLC }\end{array}$ & $\begin{array}{c}\text { DAR by } \\
\text { HIC }\end{array}$ & $\begin{array}{c}\text { Free thiol per antibody } \\
\text { by Ellman's assay }\end{array}$ \\
\hline 1 & 2.15 & 3.7 & 3.9 & 3.8 \\
2 & 2.35 & 4.1 & 4.4 & 4.4 \\
3 & 2.55 & 4.4 & 4.8 & 4.5 \\
4 & 2.75 & 4.8 & 5.0 & 5.1 \\
5 & 2.95 & 4.9 & 5.3 & 5.3 \\
\hline
\end{tabular}

a. Assuming DAR based on reduced antibody intermediate analysis.

\section{Results and Discussion}

The stochastic cysteine-based ADC was synthesized by a wellestablished approach (Fig. 1). ${ }^{19}$ The first step of this conjugation is the partial reduction of the antibody's intermolecular disulfide bonds with TCEP. The DAR of cysteine-based ADCs is dependent on the free thiol level in the reduced antibody intermediate, with the TCEP equivalent being the most crucial factor for DAR. ${ }^{19}$ To determine the TCEP equivalent for the scale-up sample, a titration examination was performed using five different TCEP equivalents $(2.15,2.35,2.55,2.75$, and 2.95 eq.) (Table 1). After completion of reduction, an in-process control (IPC) analysis of this reaction stage was conducted by Ellman's assay to determine the free thiols per antibody. Prior to Ellman's assay, gel filtration was performed in order to remove any TCEP-related materials from potentially causing undesired side reaction with Ellman's reagent. Based on the percentage of free thiols per antibody obtained from Ellman's assay, assumed DARs were calculated (the amount of free thiols per antibody of ADC precursors corresponds with the resulting DAR values of final ADCs). The indirectly-measured DAR obtained from the Elman's assay result leverages the high reactivity that thiol groups have with (excess) maleimide compounds such as MC-VC-MMAE. ${ }^{20}$ Next, commercially available MC-VC-MMAE was conjugated to the newly formed thiols on the antibody, and the difference in free thiols measured results in the DAR determination.

Five ADCs were compared by two HPLC methods: HIC and RP-HPLC. HIC-HPLC is a method commonly used for DAR determination because it can be performed under non-denaturing conditions. ${ }^{21}$

HIC-HPLC separated five DAR species (DAR $=0,2,4,6$, and 8 ) in the chromatogram (Supporting Information, Fig. S1). From these results, average DARs were calculated. However, peak resolution was relatively insufficient and some skepticism for data accuracy lingered on. HIC analysis is sometimes problematic when it comes to quality control, due to relatively low peak resolution. ${ }^{22}$ From a peak separation perspective, RPHPLC can be considered an ideal method for DAR determination, especially for cysteine-based ADCs. ${ }^{21,22}$ The high column 
Table 2 Comparison of R\&D, GMP and our approach to producing material suitable for GLP pre-clinical studies

\begin{tabular}{|c|c|c|c|}
\hline Topic & $R \& D$ & GMP & Our approach \\
\hline Facility & Non-dedicated product areas & Dedicated product areas & Segregated product areas \\
\hline \multirow[t]{2}{*}{ Equipment } & $\begin{array}{l}\text { PM, calibration and/or } \\
\text { qualification not required }\end{array}$ & $\begin{array}{r}\text { PM, calibration and/or } \\
\text { qualification required }\end{array}$ & $\begin{array}{l}\text { PM, calibration and/or qualification required only } \\
\text { for analytical equipment }\end{array}$ \\
\hline & No defined schedules & $\begin{array}{l}\text { Routine PM, calibration and } \\
\text { qualification schedules }\end{array}$ & $\begin{array}{l}\text { Routine PM, calibration and requalification } \\
\text { schedules for analytical equipment }\end{array}$ \\
\hline \multirow[t]{2}{*}{$\begin{array}{l}\text { Manufacturing } \\
\text { and laboratory } \\
\text { controls }\end{array}$} & $\begin{array}{l}\text { Experiments and studies are } \\
\text { documented at will without } \\
\text { review }\end{array}$ & $\begin{array}{l}\text { All manufacturing operations are } \\
\text { documented contemporaneously } \\
\text { with review }\end{array}$ & $\begin{array}{l}\text { All process steps are documented } \\
\text { contemporaneously with review in a } 21 \text { CFR part } \\
11 \text { compliant ELN system }\end{array}$ \\
\hline & Raw materials are off the shelf & RM control strategy in place & RMs are qualified and released prior to use \\
\hline $\begin{array}{l}\text { Analytical } \\
\text { approach }\end{array}$ & $\begin{array}{l}\text { Methods are demonstrated to be } \\
\text { suitable for their intended use }\end{array}$ & $\begin{array}{l}\text { Methods are validated and test } \\
\text { methods are documented }\end{array}$ & $\begin{array}{l}\text { Methods are approved and documented depending } \\
\text { on the maturity of the product lifecycle }\end{array}$ \\
\hline
\end{tabular}

temperature and acidic mobile phases can enhance peak separation and improve peak resolution. In this present study, RP-HPLC provided six peaks derived from antibody fragments (unconjugated light chain (LC 0), conjugated light chain by 1 MMAE (LC 1), unconjugated heavy chain (HC 0), conjugated heavy chain by 1 MMAE (HC 1), conjugated heavy chain by 2 MMAEs (HC 2), and conjugated heavy chain by 3 MMAEs (HC 3)). These six peaks separated well, demonstrating reliable DAR results (Fig. S2, SI), however both HIC-HPLC and RPHPLC were used for comparison in this study.

Slightly different results were obtained for DAR determination using the three different analytical methods, but the trend between TCEP equivalents and DAR results were comparable (Table 1). In this investigation, the relationship between TCEP equivalents and DAR was examined with limited margin, but TCEP dependency for DAR reflected slight differences for each batch. The results indicated high reactivity of TCEP for disulfide bond cleavage. DAR from RP-HPLC was slightly lower than HIC results in all investigations, which was also observed in our previous studies for site-specific ADCs. ${ }^{8}$ In many cases, HIC does not provide sufficient peak separation including positional isomers because this native analysis requires highly concentrated salt in the mobile phases which can affect the peak separation. ${ }^{22}$ The introduction of organic solvents like isopropyl alcohol (IPA) sometimes can improve peak shape, and in our previous study mobile phases that included IPA provided better resolution than organic solventfree running conditions, ${ }^{8}$ however, that may not be sufficient in this present study. On the other hand, RP-HPLC provides higher resolution than HIC due to sample pretreatment under denaturing and reducing conditions. The relatively high temperature and denaturing pretreatment with a combination of urea and TCEP have potential risks for degradation of the ADC species that may result in lower DAR values. ${ }^{22}$ Ellman's assay only provides assumed DAR based on reduced antibody intermediate analysis because Ellman's reagent reacts with free thiol functional groups. These characteristic differences among the three analyses generated slightly different DAR results.

Next, we attempted to apply GMP strategy based on FDA GLP requirements ${ }^{23}$ for cysteine-based ADC preparation. To reduce risk of cross-contamination, all conjugation and formulation activities were performed in the Process Development laboratory (PD) which is segregated from other GMP laboratories. After formulation, analytical activities were performed in a GMP regulated laboratory, which include GMP regulated equipment. GMP regulated areas and equipment are routinely maintained, calibrated and, if required, qualified.

Raw materials used for conjugation and formulation activities are GMP grade and conform to guidelines for manufacturing,

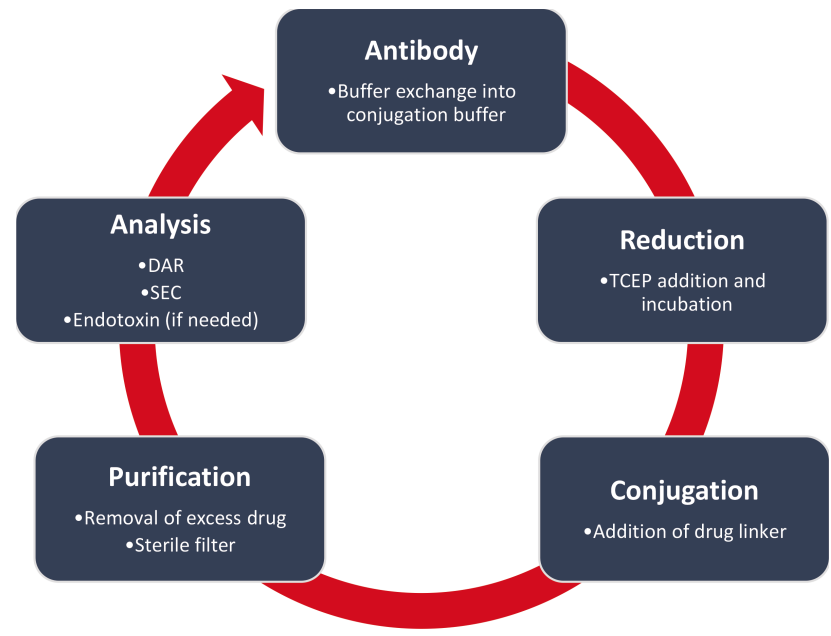

Fig. 2 Flowchart to prepare cysteine-based ADC for good laboratory practice material.

testing and quality. In-house raw materials are quarantined, sampled, tested and inspected. Results are entered into an Enterprise Resource Planning (ERP) software which aids in material control and lot release by the quality unit after the raw materials are qualified.

Proper documentation of all activities, including data collection, were captured contemporaneously on a pre-approved batch record via electronic laboratory notebook (ELN). The information and data recorded is verified by a witness and reviewed by the quality unit at gating steps within the batch record. Final review of the batch record is performed by PD and the quality unit; at the time of publication, any corrections and/or deviations were addressed.

Formulation methods used for testing and analysis were not qualified but were established to be suitable for their intended use. Analytical methods were created, reviewed and proceduralized after approval. Sampling was taken as defined in the pre-approved batch record. The corresponding data was entered into the ELN for traceability and used to create a certificate of testing. This information is reviewed by PD and the quality unit and guided the release of the material produced. The summary of the GMP strategy explaining the difference among lab scale, traditional GMP strategy and our approaches are shown in Table 2.

Finally, four hundred milligrams of ADC for use in GLP studies was conducted by the following GMP strategy (Fig. 2).

On the basis of the previous TCEP investigation described in 
Table 3 Certificate of testing for final ADC material

\begin{tabular}{lc}
\hline \multicolumn{1}{c}{ Target ADC } & Trastuzumab-MMAE \\
\hline DAR by RP-HPLC & 4.4 \\
DAR by HIC & 4.9 \\
Monomer by SEC, $\%$ & 99.2 \\
Endotoxin concentration/EU mL & -1 \\
Yield, $\%$ & $<0.100$ \\
\hline
\end{tabular}

Table 1, we aimed to make a stochastic ADC with a target DAR of 4.4. To determine the TCEP equivalents, the scale gap was required to be considered. For the small-scale investigation in Table 1, we used the shaker for conjugation. However, we identified a glass reactor vessel with a stir bar as the suitable equipment for over hundred milligram scale ADC production. This stirring system is a well-established mixing equipment, which enables the later application for kilogram-scale ADC preparation. ${ }^{24}$ We intentionally lowered the TCEP equivalent (2.35) for scale up conjugation due to the stirring efficiency of this system.

After purification and final sterile filtration of the resulting ADC, a certificate of testing (COT) was created, summarizing the analyses using the validated equipment (Table 3 ).

DAR of the scale-up batch was higher than the small-scale batch as expected. Due to the high mixing efficiency of the glass vessel reactor, TCEP reduction effectively cleaved the disulfide bond of the antibody without any side reactions..$^{18,25,26}$ IPC analysis utilizing Ellman's assay also supported these DAR results described in (Table S1, SI). Therefore, the difference in DAR from batch to batch was confirmed to be derived from the TCEP reduction step. Product yield, concentration, aggregation results (Fig. S3, SI) and endotoxin (Fig. S4, SI) were also significant for this GLP suitable material.

\section{Conclusion}

The analytical comparison for a single batch ADC produced by cysteine type conjugation was successfully conducted. The trend of increasing DAR corresponding to increasing TCEP equivalents was verified by three different methods, although a slightly different DAR value $(0.2-0.4)$ was also observed. Our present study indicated that all three analyses are useful, but a combination of them is recommended to obtain an accurate DAR value because DAR discrepancy was observed among these assays. To address this discrepancy, two approaches are ongoing.

Chemical site-specific conjugation has the ability to streamline analysis due to its simple composition. Several well-designed site-specific technologies have recently been reported, ${ }^{17,27-30}$ with some already applied to large-scale ADC synthesis. ${ }^{18}$ Another reasonable solution to simplify ADC analysis is to use homogenous ADCs, which can be obtained by preparative HIC chromatography that can separate DAR species. ${ }^{31}$ Purified DAR ADC analysis provides clear DAR results with no significant differences using six analytical methods, as shown in our previous report. ${ }^{8}$

To establish an ideal analytical approach for characterizing ADCs, many tasks and challenges remain. Several unique analytical approaches aimed at versatile ADC characterization were recently published including sensitive immunoassay, ${ }^{32}$ sample preparation for intact-MS analysis ${ }^{33}$ and preparative HPLC. ${ }^{34}$ However, we are confident that this publication can serve as a useful guide to start pre-clinical process development of antibody conjugates.

\section{Acknowledgements}

The authors wish to thank our colleagues from Ajinomoto BioPharma Services, Inc., as follows: Mr. Shawn Littrell and Mr. William Wittbold for technical assistance for GLP study; Dr. Wen Chen and Mr. Keenan Moi for technical assistance with the ADC analysis; Mr. David Correa, Mr. Steve Folio, Mr. Darwin Richardson, Mr. Bert Barbosa, and Dr. Tatsuya Okuzumi from Ajinomoto Co., Inc. for many helpful discussions and suggestions in this study.

\section{Supporting Information}

This material is available free of charge on the Web at https:// www.jsac.or.jp/analsci/.

\section{References and Notes}

1. M. Abdollahpour-Alitappeh, M. Lotfinia, T. Gharibi, J. Mardaneh, B. Farhadihosseinabadi, P. Larki, B. Faghfourian, K. S. Sepehr, K. Abbaszadeh-Goudarzi, G. AbbaszadehGoudarzi, B. Johari, M. R. Zali, and N. Bagheri, J. Cell Physiol., 2019, 234, 5628.

2. J. M. Lambert and A. Berkenblit, Annu. Rev. Med., 2018, 69, 191.

3. R. Lyon, Drug Discov. Today Technol., 2018, 30, 105.

4. A. Beck, V. D'Atri, A. Ehkirch, S. Fekete, O. HernandezAlba, R. Gahoual, E. Leize-Wagner, Y. Francois, D. Guillarme, and S. Cianférani, Expert. Rev. Proteomics., 2019, 16, 337.

5. K. Todoroki, T. Yamada, H. Mizuno, and T. Toyo'oka, Anal. Sci., 2018, 34, 397.

6. US Food and Drug Administration (FDA), Guidance for Industry-Monoclonal Antibodies Used as Reagents in Drug Manufacturing, https://www.fda.gov/media/72408/ download.

7. US Food and Drug Administration (FDA), Assay Development and Validation for Immunogenicity Testing of Therapeutic Protein Products-Guidance for Industry, https://www.fda.gov/media/77796/download.

8. Y. Matsuda, V. Robles, M-C. Malinao, S. James, and B. A. Mendelsohn, Anal. Chem., 2019, 91, 12724.

9. F. Debaene, A. Boeuf, E. Wagner-Rousset, O. Colas, D. Ayoub, N. Corvaïa, A. Van Dorsselaer, A. Beck, and S. Cianférani, Anal. Chem., 2014, 86, 10674.

10. L. N. Le, J. M. R. Moore, J. Ouyang, X. Chen, M. D. H. Nguyen, and W. J. Galush, Anal. Chem., 2012, 84, 7479.

11. D. Firth, L. Bell, M. Squires, S. Estdale, and C. McKee, Anal. Biochem., 2015, 485, 34.

12. M. Källsten, R. Hartmann, K. Artemenko, S. B. Lind, F. Lehmann, and B. Bergquist, Analyst, 2018, 143, 5487.

13. Y. Matsuda, C. Clancy, Z. Tawfiq, V. Robles, and B. A. Mendelsohn, ACS Omega, 2019, 4, 20564.

14. P. D. Senter and E. L. Sievers, Nat. Biotechnol., 2012, 30, 631.

15. H. Tilly, F. Morschhauser, N. L. Bartlett, A. Mehta, G. Salles, C. Haioun, J. Munoz, A. I. Chen, K. Kolibaba, D. Lu, M. Yan, E. Penuel, J. Hirata, C. Lee, and J. P. Sharman, Lancet Oncol., 2019, 20, 998. 
16. Z. Tawfiq, N. C. Caiazza, S. Kambourakis, Y. Matsuda, B. Griffin, J. C. Lippmeier, and B. A. Mendelsohn, ACS Omega, 2020, 5, 7193.

17. K. Yamada, N. Shikida, K. Shimbo, Y. Ito, Z. Khedri, Y. Matsuda, and B. A. Mendelsohn, Angew. Chem., Int. Ed., 2019, 58, 5592.

18. Y. Matsuda, K. Yamada, T. Okuzumi, and B. A. Mendelsohn, Org. Process Res. Dev., 2019, 23, 2647.

19. S. O. Doronina, B. E. Toki, M. Y. Torgov, B. A. Mendelsohn, C. G. Cerveny, D. F. Chace, R. L. DeBlanc, R. P. Gearing, T. D. Bovee, C. B. Siegall, J. A. Francisco, A. F. Wahl, D. L. Meyer, and P. D. Senter, Nat. Biotechnol., 2003, 21, 778.

20. G. T. Hermanson, "Bioconjugate Techniques", 3rd ed., 2013, Elsevier, London, 240 - 246.

21. S. Fekete, J.-L. Veuthey, A. Beck, and D. Guillarme, J. Pharm. Biomed. Anal., 2016, 130, 3.

22. V. D'Atri, R. Pell, A. Clarke, D. Guillarme, and S. Fekete, J. Chromatogr. A, 2019, 1586, 149.

23. Title 21 CFR Part 58 Good Laboratory Practice for Nonclinical Laboratory Studies, 18 February 2004.
24. B. Stump and J. Steinmann, Methods Mol. Biol., 2013, 1045, 235.

25. D. E. Shafer, J. K. Inman, and A. Lees, Anal. Biochem., 2000, 282, 161.

26. J. C. Han and G. Y. Han, Anal. Biochem., 1994, $220,5$.

27. Y. Matsuda, M. C. Malinao, V. Robles, J. Song, K. Yamada, and B. A. Mendelsohn, J. Chromatogr. B, 2020, 1140, 121981

28. S. Sakamoto and I. Hamachi, Anal. Sci., 2019, 35, 5.

29. K. Tsuchikama and A. An, Protein \& Cell, 2018, 9, 33.

30. K. Maruyama and M. Kanai, Chem. Lett., 2019, 48, 1421.

31. C. L. Becker, R. J. Duffy, J. Gandarilla, and S. M. Richter, Methods Mol. Biol., 2019, 2078, 237.

32. Y. Zhang, S. Zhou, X, Chang, and X. Tao, Anal. Sci., 2019, 35, 1291.

33. M. K. Sharma, P. Dhakne, S. NN, P. A. Reddy, and P. Sengupta, Anal Sci., 2019, 35, 1069.

34. Y. Matsuda, M. Leung, T. Okuzumi, and B. A. Mendelsohn, Antibodies, 2020, 9, 16. 